\title{
VIRTUAL COMPOSITE - AN EFFECTIVE TOOL OF RESEARCH
}

\author{
Miloslav Kosek \\ Technical University of Liberec, Halkova 6, 46117 Liberec 1, Czech Republic, \\ e_mail: miloslav.kosek@vslib.cz
}

Abstract: The paper deals with analysis of the structure of textile composite. The quasiperiodic yarn shape, as basic structure unit, is modelled by the Fourier series that allows high flexibility, for example very effective approximation. Amplitudes and phase constants in Fourier series exhibit the distribution very similar to normal one. By the use of random number generators, virtual yarns can be generated and virtual composite realised from them. The structures are effectively visualised by a virtual reality system. The method of visualisation allows the immediately use of finite element method to predict the properties of virtual composite. Efficient model of virtual composite saves a lot of expensive experimental work.

Key words: Textile composite, composite structure, image processing, Fourier series, discrete Fourier transform, 3D imaging, virtual reality, statistics distributions, finite element method

\section{INTRODUCTION}

Composites are new perspective materials because of their unusual mechanical properties. An important group of them are composites reinforced by a two-dimensional fabrics - textile composites. They are produced by a relatively simple technology. Several layers of woven cloth are impregnated with a suitable resin, placed one another, pressed and heated in a specified regime. The cloths, or yarns creating the cloths, are termed reinforcement and the resin is known as a matrix in composite terminology.

The original version of this chapter was revised: The copyright line was incorrect. This has been corrected. The Erratum to this chapter is available at DOI: 10.1007/978-0-387-35492-7_50 
The basic composite feature, irrespective of its type and realisation, is that composite properties are not a sum or another analytical function of individual component properties, reinforcement and matrix, for example. Also the composite structure has a strong effect on its properties. This is a reason of extensive study of the composite structure. If the structure is known with a satisfactory accuracy, the composite can be analysed, for example by the finite element method (FEM), and its properties can be predicted from suitable models.

The three-dimensional (3D) structure of textile composite is determined by yarns forming the reinforcement. This structure during composite technological processing changes from almost regular ideal to quasi-regular real structure. A very convenient way of composite structure study are images of cross-sectional cuts made by standard metallographic technique. The yarn shape can be derived from the image. Among many ways of real yarn shape description, the most suitable appears the Fourier series.

By such an approach we get only random specific samples of quasiregular real structure. A high degree of uncertainty is typical for this method. Therefore the information allows only to create a model of real composite structure, which is termed a virtual composite. Statistical methods should be a base for such model. The paper presents preliminary results necessary to realisation of the virtual composite.

\section{YARN SHAPE}

Yarn is a basic unit of the textile composite, therefore composite modelling should start by yarn analysis. The first step of such analysis is yarn visualisation. As the cross-sectional cuts were long and narrow, their parts were scanned by LUCIA imaging systems and then the whole image was composed form partial ones. Our original HW and SW was used [1]. An example of typical image is in Fig. 1. Yarns, making the reinforcement, are visible by human eye, but not by a computer, therefore the yarn axis coordinate reading was made manually.

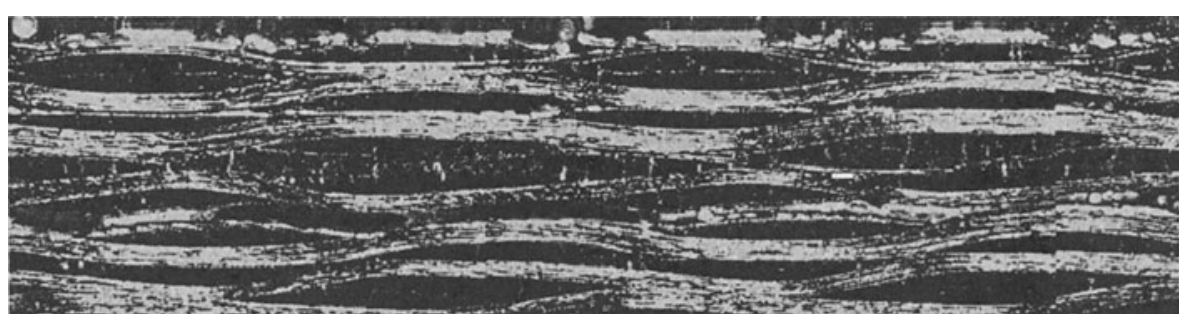

Figure 1. Image of composite cut 
The second step is yarn analytic description. There is a lot of possibilities how to approximate yarn axis: polynomial [2], splines [3], Fourier series [4]. We preferred the use of Fourier series, as all its parameters have well defined interpretation and different level of approximation can be made by selecting lower or higher number of parameters. If the yarn axis is given as the function $y(x)$ defined on the cut of length $L$, the most suitable Fourier series has the form

$$
y(x)=\frac{A_{0}}{2}+\sum_{\mathrm{n}=1}^{N} A_{\mathrm{n}} \sin \left(2 \pi n \frac{x}{L}+\varphi_{\mathrm{n}}\right)
$$

where $A_{\mathrm{n}}$ and $\varphi_{\mathrm{n}}$ are the amplitude and phase constant of the $n$th harmonics, respectively. They were obtained by the discrete Fourier transform (DFT) of the axis points get manually in the previous step.

The use of Fourier series has other advantage - the yarn axis can be described ether in the co-ordinate or wave domain as it is typical in electrical engineering. The co-ordinate domain is the usual graphical description while the wave domain consists of amplitude and phase constant spectrum. Both the forms are demonstrated in Fig. 2 and 3. Only amplitude spectrum is shown for simplicity in Fig. 3. It must be stressed that both the forms contain the same amount of information, but the information is presented in different ways. Some features are pronounced in co-ordinate domain and others in wave domain.

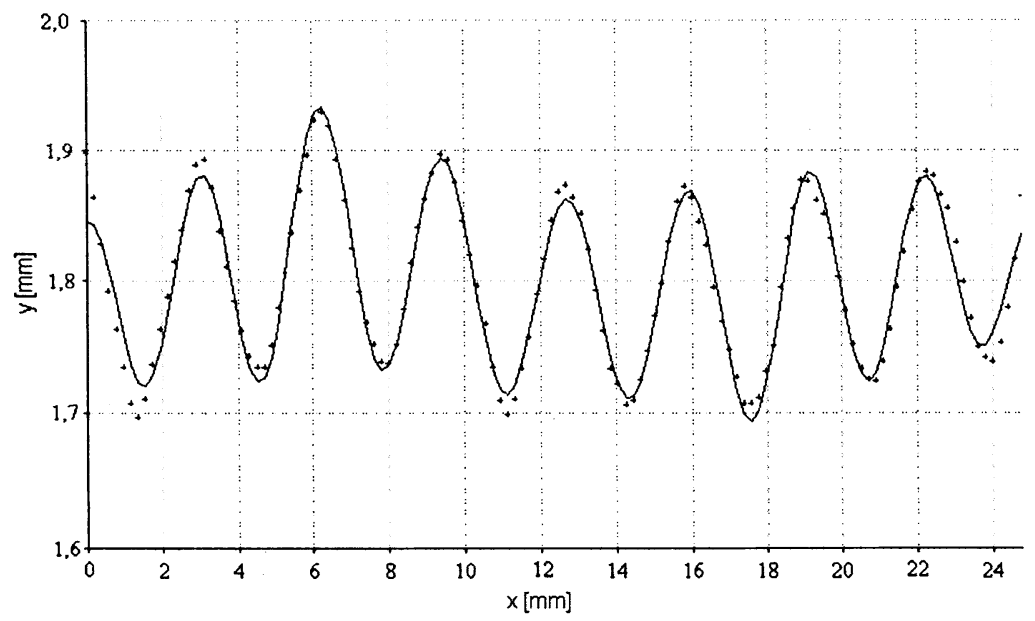

Figure 2. Yarn axis presentation in co-ordinate domain

It is evident from randomly selected yarn shape in Fig. 2 that the ideal sinusoid changes considerably during processing. Fig. 2 also illustrates the 
possibility of approximation. Only seven highest harmonics of spectra (see Fig. 3) were used and the agreement with experiment is satisfactory. Important harmonics have simple and straightforward interpretation. The highest, for example $m$ th harmonics, determines the period, or wavelength, $\lambda=L / m$. Typically $m=7$. Harmonics near to maximum are responsible for the deviation from an ideal sinusoid shape. Low harmonics determine the distortion of the layer from its initial plane shape, see sinusoid axis in Fig. 2.

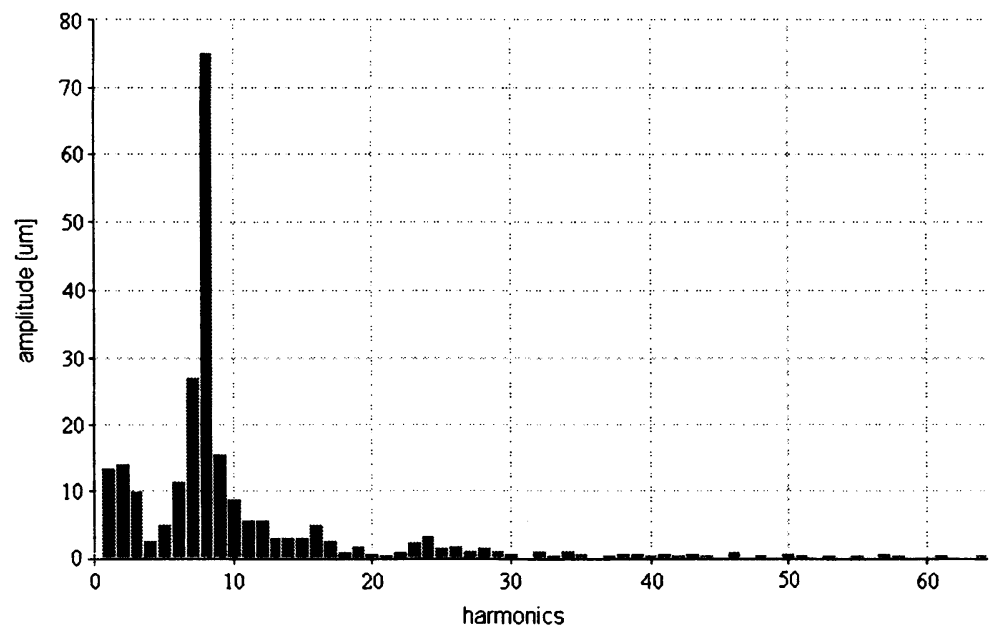

Figure 3. Amplitude spectrum of yarn - wave domain

If each yarn in the cut is approximated, we get an analytic description of the cut, which is probably the maximum information that can be get from the cut image. An example of the analytically described cut is in Fig 4. We see that yarns differ one another.

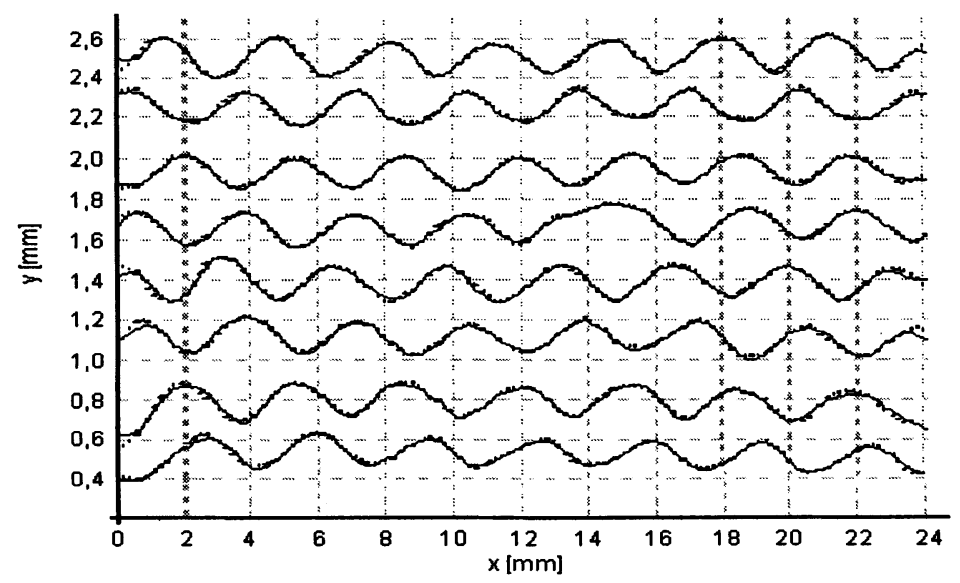

Figure 4. Approximated yarns in composite cut 


\section{YARN STATISTICS}

The method of random cuts yields yarns that are only a small random representation of all the yarns. Their parameters should be processed statistically. As a result of extended experimental work on plane weave composite, 96 samples of each harmonics were obtained. Since the amount of data is relatively low for statistical processing, non-standard procedures were used in parallel with standard ones.

As it is clear from equation (1), each harmonics is done by the amplitude and phase constant. Therefore the statistic parameters of both the amplitude and phase must be found. Only a few statistic parameters are important for us: the type of distribution and, if the distribution was found, its mean value and standard deviation. As for the type of distribution, we predict, from analysis of composite technology, that amplitudes exhibit normal and the distribution of phase constants is uniform.

Results of standard statistical SW were checked by our own programs and similar conclusions from all the systems were obtained: the phase constant distribution is uniform for all harmonics, as we expected. However, in contradiction to our prediction, normal distribution of amplitudes was found only in few cases. About $90 \%$ of harmonics exhibited the Weibull distribution. For completeness, in few cases also a log-normal distribution appeared and no other distribution was detected. All the distributions are subject on any statistics textbook.

As for statistical parameters, typically values of the variation coefficient of amplitudes is about $30 \%$. This means a large dissipation of important amplitudes from yarn to yarn and large variation of structure from sample to sample. The parameters depending on the structure should vary roughly in the same extent.

\section{COMPOSITE MODELING}

As the composite exhibits $3 \mathrm{D}$ relatively complicated structure, the computer graphics should be used for its modeling. There is a lot of methods of computer graphics which are applicable to the structure visualization. However, since final users should be textile related specialists, simple and efficient solution is necessary. As an ideal approach the Virtual Reality Modeling Language (VRML) was found for this case, since it allows many forms of structure inspection and the structure description is in a form of text file that can be quite complicated, of course.

Basic unit of composite structure is the yarn. Since its cross-section does not change, the yarn can be effectively modeled by a set of small cylinders. 
The principle is illustrated in Fig. 5. From case a) to d) the number of cylinders increases, while both their height and the distance between neighbors decrease. Quite realistic image of the yarn can be get, see part d).

Another and in some cases better choice of model element exists [5]. We preferred simple cylindrical element, since the geometrical model should be used in the FEM. Furthermore, cylinders are basic elements of VRML.

Almost all ideal 2D and 3D textile structures can be simply modeled in this way and this approach is widely used [6]. The top of structure hierarchy is $3 \mathrm{D}$ model of an ideal composite that is shown in Fig. 6.

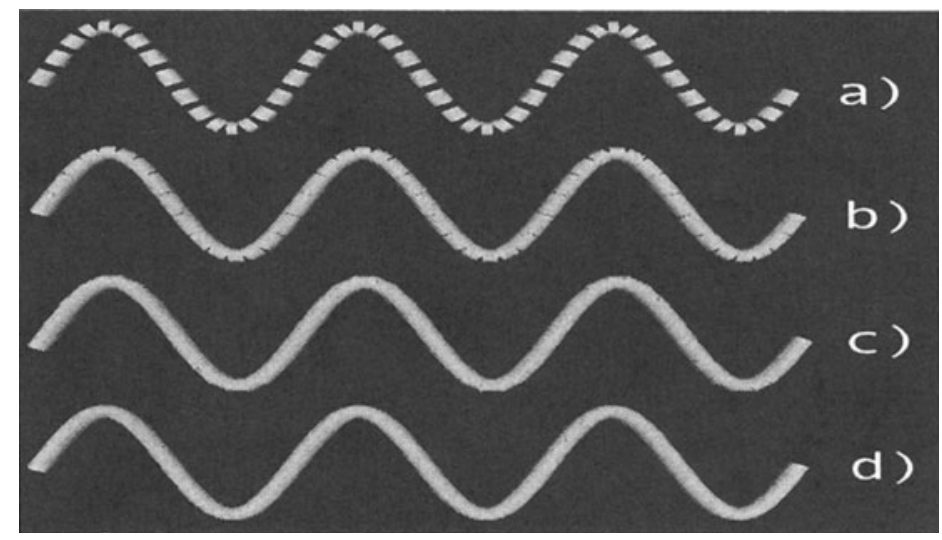

Figure 5. Principle of the yarn model

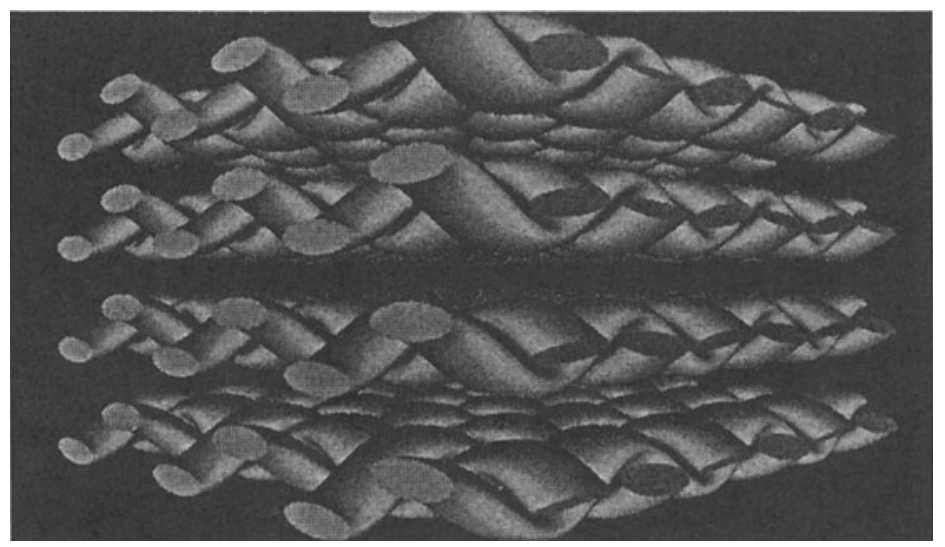

Figure 6. Ideal composite 


\section{VIRTUAL COMPOSITE}

The only knowledge on composite structure that can be get from the method of cuts, is the statistical distribution of amplitudes and phase constants and parameters of these distributions. It has been already mentioned that the most frequent amplitude distribution was the Weibull one, about $90 \%$. However, we have found that there is little difference between Weibull and normal distribution from the practical point of view in most cases. Therefore, we have used standard generators of uniform distribution to generate phase constants and MATLAB generator of normal distribution to get amplitudes. By the use of Fourier series (1) the virtual yarn can be realized from the generated random amplitudes and phase constants. An example of a virtual cut is in Fig. 7. If we compare the virtual cut with a real cut in Fig. 4, we see that the virtual structure exhibits higher irregularity than the real one.

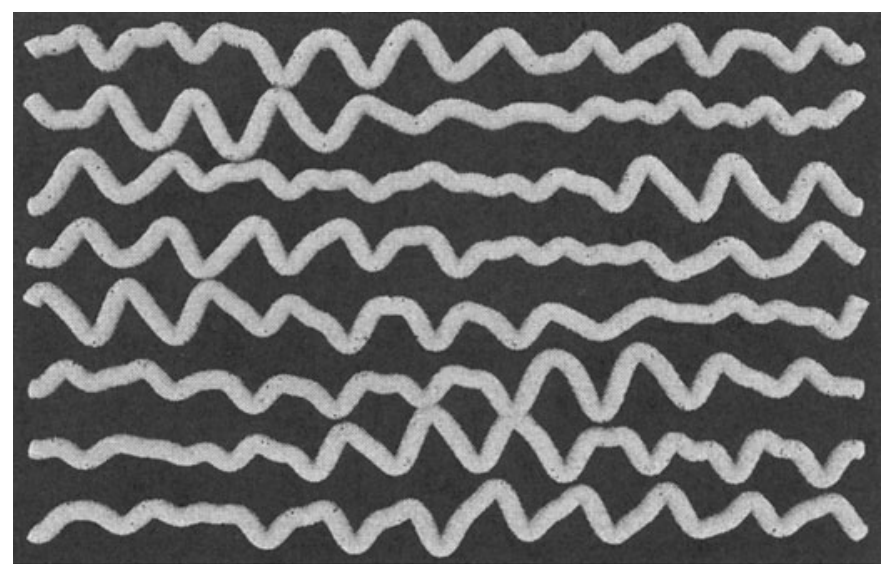

Figure 7. Cut in virtual composite

In principle the virtual textile layer and set of layers, virtual composite, can be realised by the same way. Practically, the generation of virtual textile layer is extremely difficult, since the virtual yarn intersections must be avoided. By another words, the second perpendicular set of yarns in the layer must respect a lot of constraints. This problem is not present in the case of ideal regular structure.

One of solutions of this problem supposes non-standard application of advanced methods of computer graphics to find intersections and then modify the yarn shape to avoid the intersections. Simpler solution is to find constraints from the first set of yarns and then generate random yarns of the 
second set until all the constraint condition are solved. The second solution is in progress.

\section{CONCLUSION}

The key step of virtual composite realization, inspection and processing is the reliable virtual yarn generation. Results from our simple approach reveal that there are some differences between cuts of real and virtual composite. The reason of the difference is probably the improper use of statistical data. Especially the yarn shape is very sensitive to phase constants in Fourier series. In order to get a better insight into phase constant distribution, phase shifts relative to the most important harmonics (usually the $7^{\text {th }}$ or $8^{\text {th }}$ harmonics) should be treated instead of absolute phase constants. Also comparison of random amplitudes with real ones is necessary. May be, lower limits of random number generators should be applied. On the other hand the preliminary results confirm that the used method is correct, but it requires corrections and refinements.

The generation of virtual composite is a complicated task that requires a lot of non-trivial work. However, the solution of this problem has a high value. It will make possible to generate structures very similar to real ones without expensive experiments. The virtual composite structure can be analysed by standard methods, for example by FEM. Such an approach allows to predict properties of real samples with satisfactory agreement with experiment. Furthermore, limiting cases of structure, practically impossible from experiment, can be generated to find composite behaviour in such seldom and extreme situations.

In parallel to virtual composite realisation an experiment starts that will try to get a detailed structure of a few real samples of composite. The procedure is to cut off very thin layers and make the image of the surfaces. Complete 3D structure of real composite can be composed from the images and compared with the structure of virtual composites. Unfortunately, this very extensive and time consuming experiment will have at its output only few real structures. Its main result will be to verify the method used for virtual composite.

\section{ACKNOWLEDGEMENTS}

This study was supported by the Grant Agency of the Czech Republic within the grant project No. 102/00/0696. 


\section{REFERENCES}

[1] Kosek M., Myslivec M. Simple Graphical System for Determination of Actual Yarn Geometry of Textile Composites. Proc. of Spring Conference on Computer Graphics, Budmerice, Slovakia, April 28 ${ }^{\text {th }}$ - May $1^{\text {st }}$, 1999, pp. 25-26.

[2] Yurgartis S.W., Morey K., Jortner J. Measurement of Yarn Shape and Nesting in PlainWave Composites. J. Composite Sci \& Technol., 1993;1:39-50.

[3] Vopicka S., Koskova B., Glogar, P. Approximation of Actual Yarn Shape in Textile Composites Using Cubic Splines. Proc. of $3^{\text {rd }}$ Internat. Conference "Textile Science'98", Technical University in Liberec, Czech Republic, 1998; vol. 3, pp. 580-583.

[4] Kosek M., Koskova B. Analysis of yarn wavy path periodicity of textile composites using discrete Fourier transform. Proc. International Conference on Composite Engineering, ICCE/6, Orlando, 1999, pp. 427-428.

[5] Groller E., Rau R.T., Strasser W. Modelling and Visualisation of Knitewear, IEEE Trans. on Visualisation and Computer Graphics, 1995;4:302-310.

[6] Lomov S.V., Huyasmas G., Luo Y., Prodromou A., Verpoest I. Textile geometry preprocessor for meso-mechanical and permeability models of composites, Proceedings of 9th European Conference on Composite Materials (ECCM-9), Brighton, UK, June 4-7th 2000. CD-ROM. 